\title{
Acute Diverticulitis Presenting as a Pylephlebitis: Case Report
}

\author{
Miguel Urpi Ferreruela ${ }^{1}$, Joan Carles Balboa ${ }^{1}$, Clara Tellez ${ }^{2}$, Lucas Ilzarbe ${ }^{1-3}$, Laura Carot \\ Bastard $^{1-3}$ and Cristina Alvarez Urturi ${ }^{* 1-3}$ \\ $H^{1}$ Department of Gastroenterology, Hospital del Mar,Barcelona, Spain \\ ${ }^{2}$ Department of General Surgery, Hospital del Mar,Barcelona, Spain \\ ${ }^{3}$ Colorectal Neoplasms Clinical and Translational Research Group, IMIM (Hospital del Mar Medical Research Institute), \\ Barcelona, Spain \\ *Corresponding author: Cristina Alvarez Urturi, Department of Gastroenterology, Hospital del Mar; Department of \\ General Surgery, Hospital del Mar; Colorectal Neoplasms Clinical and Translational Research Group, IMIM (Hospital del \\ Mar Medical Research Institute), Barcelona, Spain
}

\section{ARTICLE INFO}

Received: 彗 September 01, 2020

Published: 豐 September 09, 2020

Citation: Miguel Urpi Ferreruela, Joan Carles Balboa, Clara Tellez, Lucas Ilzarbe Laura Carot Bastard, Cristina Alvarez Urturi. Acute Diverticulitis Presenting as aPylephlebitis: Case Report. Biomed J Sci \& Tech Res 30(2)-2020. BJSTR. MS.ID.004913.

\section{ABSTRACT}

A 47-year-old male suffered from high fever, abdominal pain and vomiting of 4 days of evolution. Blood tests showed high inflammatory parameters and abdominal CT revealed intrahepatic portal gas, partial thrombosis of intrahepatic left portal vein and sigmoid thickening with a possible foreign body. Broad spectrum antibiotic and anticoagulation were initiated, and control CT showed resolution of the intrahepatic pneumatosis, with no other changes. Treatment was maintained with a suspected diagnosis of a pylephlebitis secondary to an intraabdominal infection with good evolution.

\section{Case Presentation}

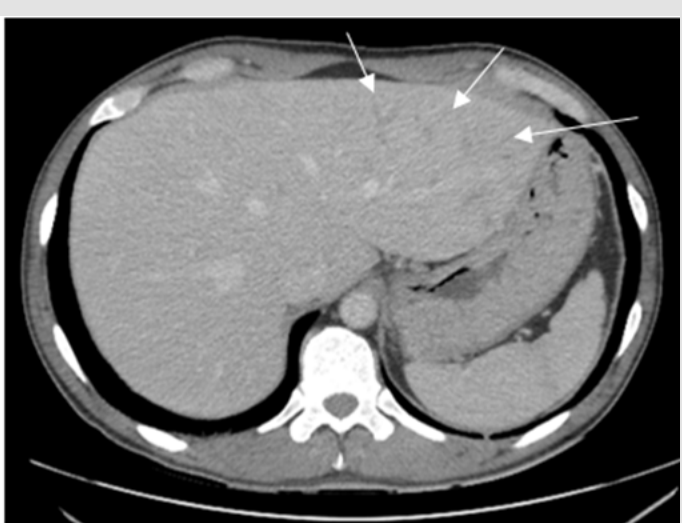

Figure 1: Computed Tomography, portal venous phase (first 24h). Periportal edema in the left hepatic lobe (marked by arrows).
The patient is a 47-year-old male, active smoker, with past medical history of human immunodeficiency virus (HIV) on active antiretroviral therapy (Triumeq)and an undetectable viral load in the last control He came to the emergency room reporting high fever $\left(>39^{\circ} \mathrm{C}\right)$ associated withabdominal pain for the last 4 days. With a first diagnosis of probable COVID-19, and awaiting the ambulatory results from a first consultation, he consulted again due to the worsening of his condition even after taking the antithermic treatment.At his arrival he presented fever with shivering, bilious vomiting and nonspecific abdominal pain. On physical examination, the patient had a temperature of $38^{\circ} \mathrm{C}$, heart rate of 123 , blood pressure of $95 / 64 \mathrm{~mm} \mathrm{Hg}$, and breathing at a rate of 16 with $97 \%$ oxygen saturation on room air. He was completely alert and oriented and he had tenderness to palpation in the left lower quadrant and in the periumbilical area. Rest of his exam was essentially normal. After the previous SARS-COV-2 test's results 
being negative, a second rapid test came negative too. The blood test revealed leukocytosis with neutrophilia $(21000 / \mu \mathrm{L} ; \mathrm{N} 86 \%)$ reactive C protein of $24 \mathrm{mg} / \mathrm{dl}$, procalcitonine of $10 \mathrm{ng} / \mathrm{ml}$, and a mild liver function alteration (total bilirubin $0.6 \mathrm{mg} / \mathrm{dl}$, AST/ALT 34/42 U/L, ALP 247 U/L)(Figures 1\&2).

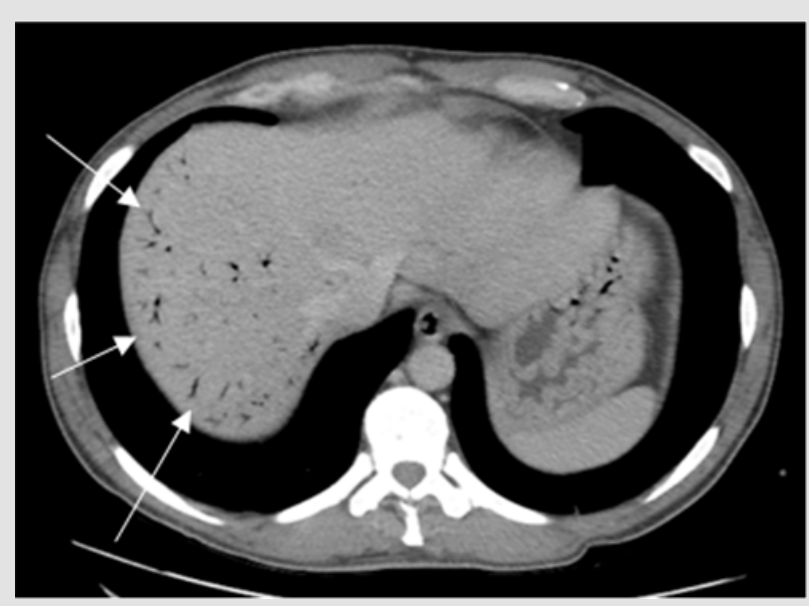

Figure 2: Contrast-enhanced CT (at 48h).Portal intrahepatic pneumatosis, with predominant peripherical location in the right hepatic lobe (marked by the arrows).

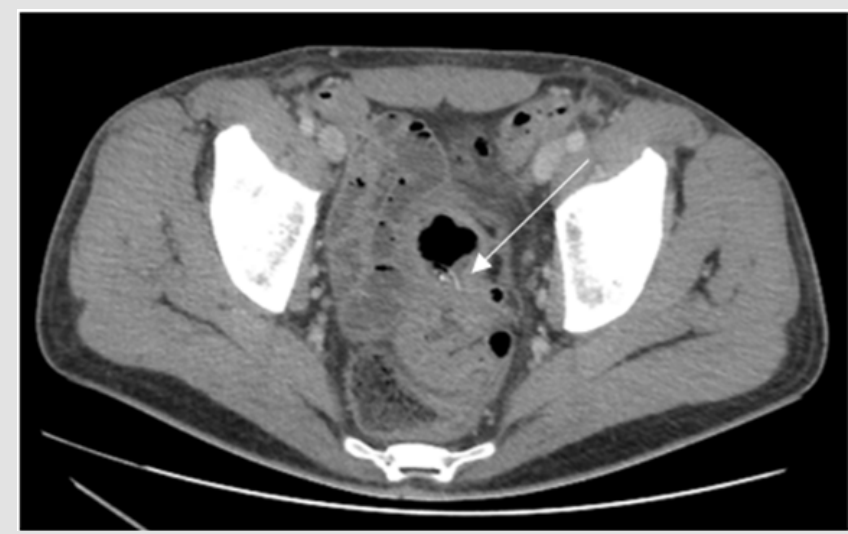

Figure 3: Contrast-enhanced CT (at 48h). Sigmoid diffuse thickening associated with sigmoid diverticulosis. Lineal filiform foreign body probably emplaced in a diverticulum, with bone density (marked by the arrow).

Under the suspicion of an infective intraabdominal process an abdominal contrast-enhanced computed tomography (CT) was done. The CT revealed an undefined area of hypo-attenuation in segment 4, periportal edema in the left hepatic lobe (LHL), and many sigmoid diverticulosis with a significant thickening of the sigmoid wall, with no clear signs of diverticulitis. Having these findings, the patient was admitted Intensive Care Unit and intravenous antibiotics were started (imipenem and linezolid) for a broad-spectrum abdominal coverage.A CT was repeated after $24 \mathrm{~h}$, finding resolution of the periportal edema (LHL) and an important quantity of intrahepatic portal gas, and a partial thrombosis in the left intrahepatic portal vein, suggesting a focal site of pylephlebitis, with continuity to the previously citated area in segment 4 . About the sigmoid thickening, no changes were identified, except from a diverticulum with a lineal possible foreign body (bone density), which was suspected as a possible firs site of intraabdominal infection.Therefore, anticoagulation was initiated with low molecular weight heparin (LMWH)(Figures 3\&4).

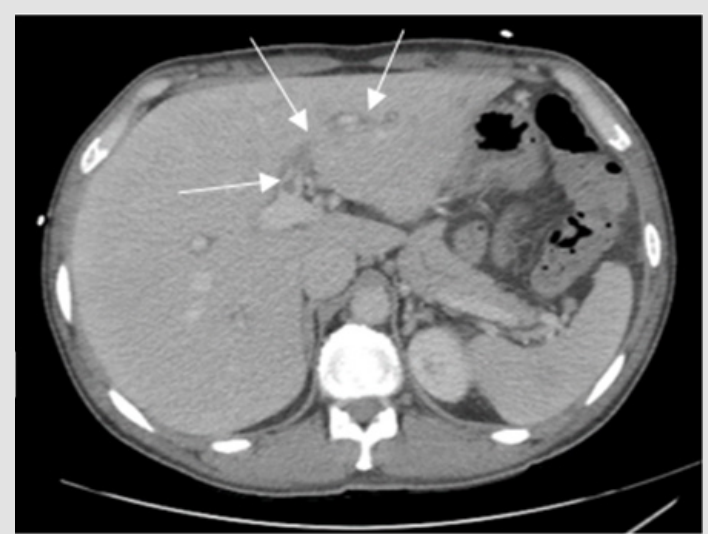

Figure 4: Contrast-enhanced CT (at 72h). Disappearance of intrahepatic pneumatosis, and persistence of intrahepatic left portal vein thrombosis (marked by the arrows), suggesting pylephlebitis.

In order to improve the diagnosis of the primary intraabdominal process (foreign body vs. acute diverticulitis), another CT control was made (after $48 \mathrm{~h}$ from the first one), showing resolution of the intrahepatic pneumatosis, confirming the persistence of the left intrahepatic portal vein thrombosis and no significant changes at the sigma level. The patient followed a good evolution after $48 \mathrm{~h}$ of antibiotic treatment, with resolution of abdominal mild pain, fever, and blood test inflammatory parameters improvement. The result from initial blood cultures being positive to S. Fusobacterium, Peptoniphilusasaccharolyticus, and coagulase negative Staphylococcus. To complete the study, a colonoscopy was undergone, which identified multiple diverticula in the sigmoid colon and identified local suppuration in one of them which suggested local acute diverticulitis. No foreign body was found. The days after, his condition got better thus was transitioned to oral antibiotic and discharged with outpatient follow-up with general surgery.In addition, he was instructed to maintain anticoagulant treatment for at least 3 months until the appointment of General Surgery.

\section{Discussion}

Pylophlebitis is an uncommon entity consistent in infective thrombosis of the portal vein, it is usually associated to an intraabdominal septic process, or secondary to surgeries, traumatisms or hypercoagulation states. The infective process affects the endothelium originating thrombosis than migrate through portal drainage and affect its territory, and its intrahepatic branches being able, if untreated, to originate hepatic abscesses, septic shock, hepatic dis- 
function, progression of the thrombosis to the mesenteric territory, secondary intestinal ischemia,etc. [1,2].The most frequent cause reported is diverticulitis (30\%), followed by appendicitis (19\%), inflammatory bower disease, pancreatitis, and others [1].In this case, the cause was initially suspected to be an intra-abdominal sepsis located in the sigma, with secondary suspicion after colonoscopy of a focal diverticulitis.The symptoms are totally unspecific being the more common ones, fever (86\%) and abdominal pain (82\%). It is also frequently accompanied of bacteraemia, with blood cultures positive in a high percentage (up to $80 \%$ in some series) [1,2]. The bacteraemia associated is frequently polymicrobial. Anaerobes represented by Bacterioides spp., are the most frequent identifiable cause, being E.Coli the second, and Streptococcus spp. the third one group $[1,3,4]$. Bacteraemia by Fusobacterium, as in this case, has also been found in similar cases [5].

As for laboratory findings, leukoctosis is the most common one, also liver function alteration with increasement of transaminases more frequent than alkaline phosphatase, glutamyl transfrerase, or bilirubin [1]. In this case only with an alteration of ALP and transaminases was detected.Recently the incidence of Pylophlebitis has increased, probably due to the availability of image testing. Diagnosis can be made in a compatible clinical case, with a radiological confirmation of thrombosis. This can be achieved, either by ecography, with doppler mode, than allow to evaluate venous flow, thrombosis, and clot recanalization, or by CT (being the best diagnostic method), allowing to identify its extention and a primary intraabdominal cause of the pylephlebitis[1,3]. Some of the possible complications that presented the patient, were intrahepatic portal thrombosis extension, and intraportal gas, only present in $18 \%$ of cases. No liver abscess, extrahepatic extension of the thrombosis nor bowel ischemia was found [1](Figure 5).

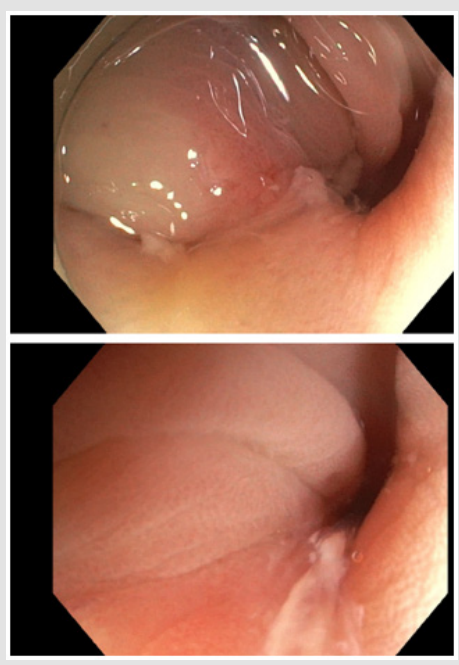

Figure 5: Colonoscopy. Sigmoid colon with multiple smallsized diverticular orifices. It checks the area exhaustively, aiming in a punctual way and $25 \mathrm{~cm}$ anal marge, the edematous mucosa with sorted material purulent in a small diverticle of $4-5 \mathrm{~mm}$.
Treatment recommended for pylophlebitis is empirical antibiotic, covering polymicrobial infections, both gram negative aerobes and anaerobes, until the results of antimicrobial susceptibility tests (from blood or surgical cultures) are available. With a total duration ranging from 4 to 6 weeks [1,4].In most scenarios, surgery is not required, but in some case, it may be necessary to control de primary focus of infection. The necessity of anticoagulation is not well established. It is recommended if the thrombosis extends to mesenteric territory, there is an hypercoagulation state, an infection from Bacteroides spp. is detected, thrombosis progression appears in successive controls or there is persistence of the fever or clinical signs of infection [18]. There are studies, in which early anticoagulation translates into a more rapid recanalization of the clot and less fatal events $[1,8]$. Nevertheless, there is also a lack of evidence for the recommendation of time of anticoagulation therapy.

In conclusion, pylephlebitis is an infrequent complication of all kind of intraabdominal infections, severe, and with a high morbidity and mortality. It must be suspected of, because with an early diagnosis and rapid instauration of treatment with antibiotic, anticoagulant or surgical control of the first focus if needed, are the main actions to prevent progression, further complications, and mortality.

\section{Funding}

This work was supported by grants from Grups Recerca Generalitat Catalunya (2017 SGR 00080).

\section{References}

1. Kanellopoulou T, Alexopoulou A, Theodossiades, John Koskinas, J Archimandritis (2010) Pylephlebitis: An overview of non-cirrhotic cases and factors related to outcome. Scandinavian Journal of Infectious Diseases 42(11-12): 804-811.

2. Arteche E, Ostiz S, Miranda L, P Caballero, GJ Lopez, et al. (2005) Tromboflebitis séptica de la vena porta (pileflebitis): Diagnóstico y manejo a propósito. Anales del sistema sanitario de Navarra 28(3): 417 420 .

3. Susana Pérez Bru, Carmen Nofuentes Riera, Andrés García Marín, Paloma Luri Prieto, Miguel Morales Calderón et al. (2015) Pileflebitis: una extra pero posible complicación de las infecciones intraabdominales. Cirugía y Cirujanos 83(6): 501-505.

4. Plemmons RM, Dooley DP, Longfield RN (2015) Septic thrombophlebitis of the portal vein (pylephlebitis): diagnosis and management in the modern era. Clinical infectious diseases: an official publication of the Infectious Diseases Society of America 21(5): 1114-1120.

5. Mellor TE, Mitchell N, Logan J (2017) Lemierre's syndrome variant of the gut. BMJ case reports: bcr2017221567.

6. Duffy F, Millan MT, Schoetz DJ (1995) Suppurative pylephlebitis and pylethrombosis: the role of anticoagulation. The American surgeon 61(12): 1041-1044

7. Kasper DL, Sahani D, Misdraji J (2005) Case records of the Massachusetts General Hospital. Case 25-2005. A 40-year-old man with prolonged fever and weight loss. The New England journal of medicine 353(7): 713-722.

8. Condat B, Pessione F, Helene Denninger, D Hillarie, D Valla (2000) Recent portal or mesenteric venous thrombosis: increased recognition and frequent recanalization on anticoagulant therapy. Hepatology 32(3): 466-470. 
ISSN: 2574-1241

DOI: $10.26717 /$ BJSTR.2020.30.004913

Cristina Alvarez Urturi. Biomed J Sci \& Tech Res

(C) (i) This work is licensed under Creative

Submission Link: https://biomedres.us/submit-manuscript.php

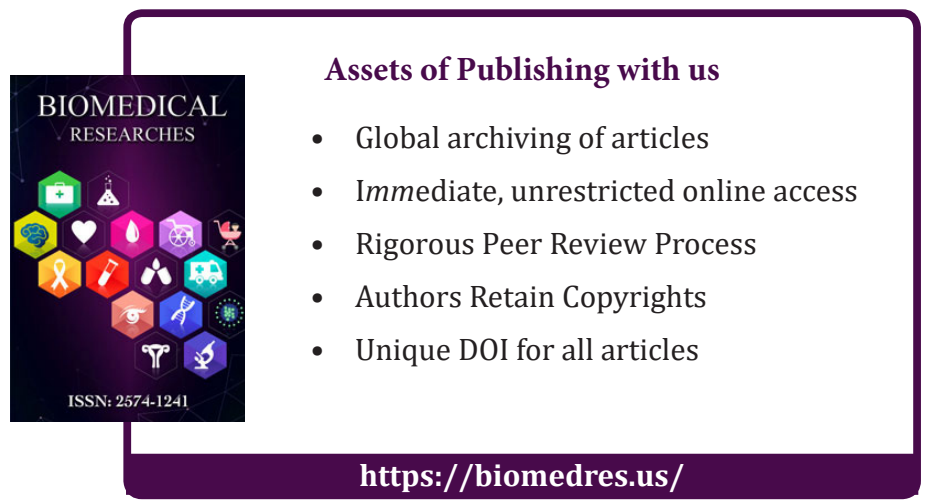

